\title{
A Reliable Prognostic Marker for Liver Dysfunction in COVID-19 Infection
}

CrossMark

\author{
Amir Anushiravani ${ }^{1}$, Bardia Khosravi ${ }^{1}$, Bahar Saberzadeh-Ardestani ${ }^{1}$, Ali Ghasemi ${ }^{2}$, Saeed Kalantari ${ }^{3}$, \\ Majid Sorouri ${ }^{1}$, Helia Mojtabavi ${ }^{1}$, Omid Ghaemi ${ }^{4}$, Amir Reza Radmard ${ }^{4}$, Amir Kasaeian ${ }^{5}$, \\ Omid Motamedi ${ }^{6}$, Hossein Poustchi ${ }^{1}$, Ali Reza Sima ${ }^{1, *}$
}

1. Digestive Disease Research Center, Digestive Disease Research Institute, Tehran University of Medical Sciences, Tehran, Iran

2. Zanjan MRI Center, Radiology Department, Vali-e-Asr Hospital, Zanjan, Iran

3. Antimicrobial Resistance Research Center, Iran University of Medical Sciences, Tehran, Iran

4. Department of Radiology, Shariati Hospital, Tehran University of Medical Sciences, Tehran, Iran

5. Hematology, Oncology and Stem Cell Transplantation Research Center, Tehran University of Medical Sciences, Tehran, Iran

6. Department of Radiology, Rasool Akram Hospital, Iran University of Medical Sciences, Tehran, Iran

* Corresponding Author:

Ali Reza Sima, M.D.

Digestive Diseases Research Institute, Tehran University of Medical Sciences, Shariati Hospital, Kargar Shomali Avenue, Tehran, Iran

Tel: + 982182415000

Fax: + 982182415400

Email: simaalireza@gmail.com

Received: 20 Nov. 2020

Accepted: 02 Jun. 2021

\section{ABSTRACT}

\section{BACKGROUND}

In December 2019, COVID-19 emerged from China and spread to become a pandemic, killing over 1,350,000 up to November 18, 2020. Some patients with COVID-19 have abnormal liver function tests. We aimed to determine the clinical significance of liver chemistries in patients with COVID-19.

\section{METHODS}

We performed a cross-sectional study of 1044 consecutive patients with confirmed COVID-19 in two referral hospitals in Tehran, Iran, from February to April 2020. All cases were diagnosed by clinical criteria and confirmed by characteristic changes in the spiral chest computed tomography (CT) and nucleic acid testing of the nasopharyngeal samples. We evaluated the association between abnormal liver enzymes or function tests and survival, intensive care unit (ICU) admission and fatty liver changes in CT scans.

\section{RESULTS}

The mean age was $61.01 \pm 16.77$ years, and $57.68 \%$ were male. Of 495 patients with elevated alanine transaminase (ALT) levels, 194 had chest CT scans, in which fatty liver disease was seen in $38.1 \%$. 41 patients $(21.13 \%)$ had moderate to severe, and $33(17.01 \%)$ had borderline fatty liver disease. Bilirubin, albumin, and partial thromboplastin time (PTT), along with other markers such as $\mathrm{HCO}_{3}, \mathrm{C}$-reactive protein (CRP), triglyceride, and length of admission, were significantly associated with ICU admission and mortality. Prothrombin time (PT), platelet count, and low-density lipoprotein (LDL) levels were also correlated with mortality. Fasting blood sugar (FBS) and $\mathrm{pH}$ were important indices in ICU admitted patients.

\section{CONCLUSION}

Liver function tests accurately predict a worse prognosis in patients with COVID-19. However, liver enzymes were only slightly increased in those who died or needed ICU admission and were not related to the fatty liver changes.

\section{KEYWORDS:}

Aminotransferase; Liver function; Liver injury; Outcome; SARS-CoV-2

Please cite this paper as:

Anushiravani A, Khosravi B, Saberzadeh-Ardestani B, Ghasemi A, Kalantari S, Sorouri M, Mojtabavi H, Ghaemi O, Radmard AR, Kasaeian A, Motamedi O, Poustchi H, Sima AR. A Reliable Prognostic Marker for Liver Dysfunction in COVID-19 Infection. Middle East J Dig Dis 2021;13:193-199. doi: 10.34172/mejdd.2021.225. 


\section{INTRODUCTION}

In December 2019, a new coronavirus (SARS-CoV-2) emerged from China and started a worldwide pandemic. ${ }^{1}$ This severe acute respiratory syndrome coronavirus-2 (SARS-CoV-2) outbreak has spread out rapidly in Iran since February 19, 2020, after the confirmation of the very first mortality case in the city of Qom. ${ }^{2}$ As of November 18,2020 , a total of 801,894 cases and 42,941 deaths due to COVID-19 have been confirmed in Iran. ${ }^{3}$

The coronavirus family includes zoonotic viruses like the Middle East respiratory syndrome (MERS) and severe acute respiratory syndrome (SARS) that can lead to respiratory and intestinal complications in both animals and humans., ${ }^{4,5}$

The primary manifestations of COVID-19 infection are respiratory symptoms. For the first time, Chen and colleagues reported that $43(43.3 \%)$ of the 99 patients with COVID-19 in Wuhan had elevated aminotransferase levels. ${ }^{6}$ Several studies have reported abnormal liver function tests in patients with COVID-19, introducing the liver as the most common extra-pulmonary damaged site among these patients. ${ }^{7}$ Besides, several studies have shown a relationship between the severity of the disease and liver damage in these patients. ${ }^{8}$ The overall prevalence of chronic liver disease on admission in patients with COVID-19 was 3\%. ${ }^{9}$ The prevalence of liver damage among mortality cases has been reported to be approximately $50.06 \%$ and $78 \% .^{10,11}$

But development, prevalence, pathophysiology, and characteristics of this liver injury are yet to be studied. ${ }^{12}$ We aimed to investigate liver chemistries and their clinical significance in patients with COVID-19.

\section{MATERIALS AND METHODS}

This was a multicenter cross-sectional study. We recruited 1119 patients with COVID-19 from February to April 2020 in two tertiary care centers in Tehran, Iran. The patients' admission date was from February to April 2020. All cases were diagnosed by clinical criteria and confirmed by characteristic changes in the spiral chest computed tomography (CT) (diagnosed by at least one diagnostic radiology specialist) and a positive reverse transcriptionpolymerase chain reaction (RT-PCR) test for SARSCoV-2. We followed our national guideline that was similar to the World Health Organization's (WHO) guideline.
All cases had symptoms such as fever or cough and met the admission criteria. Outpatient cases were not included in the study. After the exclusion of three patients with hepatitis $\mathrm{B}$, one with hepatitis $\mathrm{C}$, another one with cryptogenic cirrhosis, and 70 patients who did not have any of their liver enzymes checked, 1044 patients remained in the study. The presence of hepatitis B and $\mathrm{C}$ was diagnosed based on the serological tests that were carried out by enzyme-linked immunosorbent assay (ELISA). This study was approved by the Ethics Committee of Tehran University of Medical Sciences and was exempted from the patients' need for informed consent.

Demographic information, laboratory findings, and patients' outcomes were collected from the patients' medical records. The recorded variables were age, total bilirubin, direct bilirubin, aspartate aminotransferase (AST), alanine aminotransferase (ALT), alkaline phosphatase (ALP), albumin, partial thromboplastin time (PTT), prothrombin time (PT), $\mathrm{pH}, \mathrm{HCO}_{3}$, platelet count, C-reactive protein (CRP) level, fasting blood sugar (FBS), total cholesterol, triglyceride, low-density lipoprotein (LDL), high-density lipoprotein (HDL), fibrinogen, lactate dehydrogenase (LDH), thyroid-stimulating hormone (TSH), and length of admission. The patient's survival and intensive care unit (ICU) admission data were only available in one center.

Since laboratory tests were individualized for each patient based on clinical symptoms, all of the mentioned parameters were not available for all the cases. Abnormal liver chemistries were defined as ALT or AST levels higher than the normal range, including ALT (9-40 U/L), AST (13-40 U/L), LDH (109-245 U/L), ALP (35-100 U/L), and total bilirubin $(0.2-1.2 \mathrm{mg} / \mathrm{dL})$.

All the patients with abnormal liver chemistries were evaluated for fatty liver disease by a specialized diagnostic radiologist. In non-contrast chest CT (which was obtained as part of our national COVID-19 guideline) of patients with COVID-19 pneumonia, the upper abdominal cuts were used to evaluate fatty liver changes. The region of interest (ROI) was placed in the liver parenchyma at the areas appearing homogenous, devoid of vessels, and away from the liver capsule. The same method was applied for spleen density measurement. The average area of ROIs was $8-10 \mathrm{~cm}^{2}$ for the liver and spleen. Three ROIs for the liver (two in the right and one in the left lobe) and one ROI 
for the spleen were drawn. The mean value of three ROIs in the liver was used for hepatic density measurement. The liver density of less than 48 Hounsfield units (HU) or more than $10 \mathrm{HU}$ less than spleen density was considered as moderate to severe fatty liver. ${ }^{13}$

\section{Statistical Analysis}

Means for continuous variables were compared using independent groups $\mathrm{t}$ test when the data were normally distributed; otherwise, the Mann-Whitney test was used. We used the Chi-square test to compare the distribution of each variable between the two groups. However, when the criteria for using the Chi-square test were not met (having frequencies less than five in 2 by 2 cross-tabulation), we used Fisher's exact test instead. The categorical variables were expressed as number (\%) and compared by Fisher's exact test. Differences were considered significant at $p<$ 0.05 with a two-tailed test. All analyses were performed using Stata software version 11.

\section{RESULTS}

A total of 1119 patients with COVID-19 were enrolled. Five patients with baseline chronic liver disease and 70 patients without liver chemistries were excluded from our study. The remaining 1044 patients had a mean age of $61.01 \pm 16.77$ years, and $57.68 \%$ of them were male. We knew the outcomes (discharged or dead) of 427 patients; of them, $353(82.7 \%)$ were discharged, and 74 (17.3\%) died until the day that the data were collected. Of 405 patients, 90 patients $(22.2 \%)$ were admitted to the ICU, and the rest were in non-ICU wards. We did not have data regarding where 22 patients were admitted.

A total of 495 (47.41\%) patients had elevated ALT levels, of whom 194 had chest CT scans. Moderate to severe fatty liver disease was seen in $41(21.13 \%)$ patients.

Increased ALT or AST in patients with COVID-19 were seen in 286/1044 (27.39\%) and 495/1040 (47.59\%) patients, respectively. Most patients presented with mild abnormalities $(<80)$, and AST and ALT levels $>10 \times$ ULN (Upper limit of normal) were seen in nine and four patients, respectively. ALP was elevated in $15.07 \%$, with 50 patients having levels $>2 \times$ ULN.

The mean AST, ALT, ALP, and albumin levels had no significant correlation with liver density, $-5 \%(p=0.43)$, $-9 \%(p=0.20),-4 \%(p=0.58)$, and $4 \%(p=0.64)$, respectively (figure 1).

Higher total bilirubin, direct bilirubin, PTT, PT, CRP, triglyceride, and length of admission and lower albumin, bicarbonate, platelet count, and LDL were associated with higher mortality. Details are highlighted in table 1. Furthermore, ICU admitted patients had significantly higher total bilirubin, direct bilirubin, PTT, CRP, triglyceride, FBS, and length of admission, and lower albumin, bicarbonate, and pH. Details are shown in table 2 .

\section{DISCUSSION}

In this multicenter study, 495/1044 (47.41\%) patients had elevated ALT levels and the serum indices of liver function, including total and direct bilirubin, albumin, and PTT, along with other markers such as $\mathrm{HCO}_{3}, \mathrm{CRP}$, triglyceride, and length of admission, were significantly associated with ICU admission and mortality. PT, platelet count, and LDL levels were also correlated with mortality. FBS and $\mathrm{pH}$ were important indices in ICU admitted patients. This shows that liver function tests could accurately predict a worse prognosis in patients with COVID-19. On the other hand, liver enzymes were only slightly increased in those who died or were admitted to the ICU. Also, higher age and longer length of admission have a prominent role in predicting worse outcomes in patients with COVID-19.

Liver injury has commonly been reported in patients with previous coronaviruses (SARS-CoV and the MERS coronavirus), and liver injury was associated with their severity. ${ }^{14}$ Zhang and colleagues reported that the prevalence of pre-existing liver disease among patients with COVID-19 was between $2 \%$ to $11 \%$, and the prevalence of abnormal aminotransferases was $16-53 \%{ }^{15}$ Guan revealed that increased levels of AST were seen in $18.2 \%$ and $39.4 \%$ of patients with non-severe and severe COVID-19, respectively. Elevated ALT levels were reported in $19.8 \%$ and $28.1 \%$ of patients with non-severe and severe diseases. ${ }^{16}$ AST was elevated in $8 / 13(62 \%)$ of patients admitted to the ICU in comparison with $7 / 28$ $(25 \%)$ of patients not admitted to the ICU, as reported by Huang and co-workers. ${ }^{11}$ Another study by Wang and others also showed that patients with COVID-19 who were admitted to the ICU had a mean ALT level of 35 (U/L) and AST of 52 (U/L), which were significantly higher than non-ICU patients. ${ }^{17}$ Our study showed that 

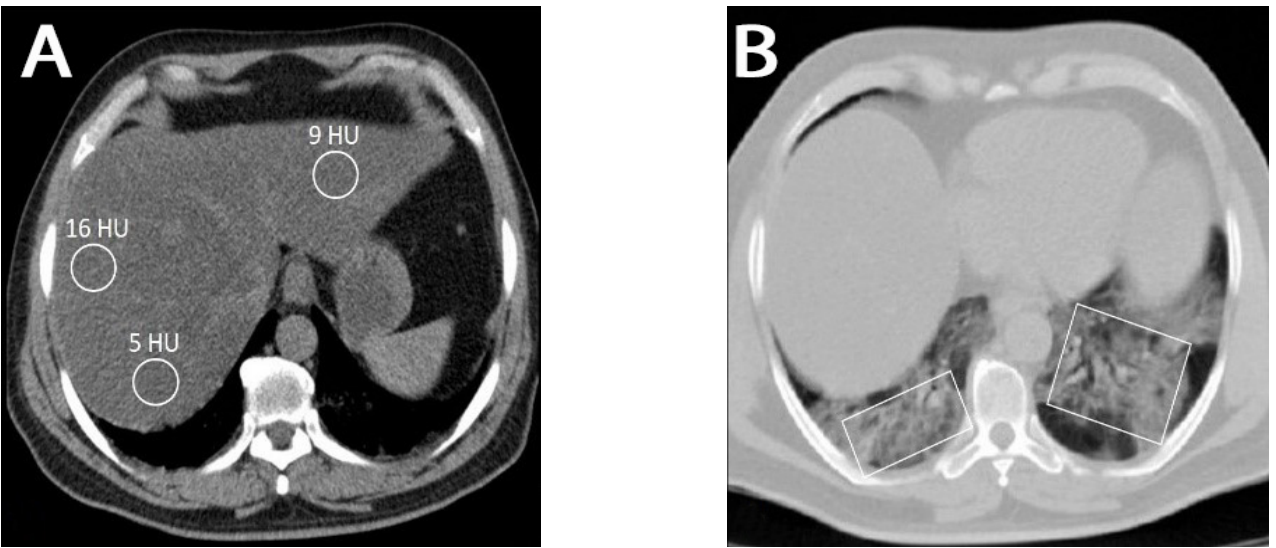

(a) Mean density of regions of interest in both hepatic lobes was 10 Hounsfield units (HU) consistent with severe fatty liver.

(b) There are bilateral patchy ground-glass opacities and early consolidations (boxes) in lower lobes highly suggestive of COVID-19 pneumonia.

Fig. 1: Non-contrast chest computed tomogram of a 52-year-old male patient with abnormal LFT, fever, cough, and myalgia without respiratory distress who had an unremarkable medical history.

Table 1: Comparison of different variables in discharged and dead patients with COVID-19

\begin{tabular}{|c|c|c|c|}
\hline Variable & $\begin{array}{c}\text { Discharged (mean/median) } \\
(\mathrm{n}=353)\end{array}$ & $\begin{array}{l}\text { Dead (mean/median) } \\
\qquad(n=74)\end{array}$ & $p$-value \\
\hline Age (year) & 59.97 & 64.81 & 0.022 \\
\hline Total bilirubin (mg/dL) & 1.27 & 2.93 & 0.002 \\
\hline Direct bilirubin $(\mathrm{mg} / \mathrm{dL})$ & 0.49 & 1.38 & 0.000 \\
\hline AST (U/L) & 58.99 & 73.34 & 0.462 \\
\hline $\operatorname{ALT}(\mathrm{U} / \mathrm{L})$ & 50.67 & 45.12 & 0.796 \\
\hline $\operatorname{ALP}(\mathrm{U} / \mathrm{L})$ & 271.84 & 310.73 & 0.520 \\
\hline Albumin (mg/dL) & 3.60 & 2.93 & 0.000 \\
\hline PTT (second) & 27.94 & 32.14 & 0.032 \\
\hline PT(second) & 16.70 & 19.07 & 0.048 \\
\hline $\mathrm{pH}$ & 7.38 & 7.36 & 0.078 \\
\hline $\mathrm{HCO} 3(\mathrm{mEq} / \mathrm{L})$ & 24.19 & 21.87 & 0.006 \\
\hline Platelet (per $\mu \mathrm{l}$ ) & 211,698 & 171,208 & 0.007 \\
\hline $\mathrm{CRP}(\mathrm{mg} / \mathrm{L})$ & 55.81 & 69.31 & 0.023 \\
\hline FBS (mmol/L) & 138 & 165 & 0.120 \\
\hline Total cholesterol (mg/dL) & 126 & 109 & 0.309 \\
\hline Triglyceride (mg/dL) & 125 & 194 & 0.020 \\
\hline $\mathrm{LDL}(\mathrm{mg} / \mathrm{dL})$ & 71 & 38 & 0.014 \\
\hline HDL (mg/dL) & 31 & 28 & 0.609 \\
\hline Fibrinogen (mg/dL) & 572 & 510 & 0.707 \\
\hline LDH (U/L) & 950 & 1168 & 0.177 \\
\hline TSH (mU/L) & 2.75 & 2.12 & 0.766 \\
\hline Length of admission (day) & 5.60 & 8.27 & 0.000 \\
\hline
\end{tabular}

ALT, Alanine aminotransferase; AST, Aspartate aminotransferase; LDH, Lactate dehydrogenase; ALP, Alkaline phosphatase; CRP, C-reactive protein; PTT, partial thromboplastin time; PT, Prothrombin time; FBS, Fasting blood sugar; LDL, Low-density lipoproteins; HDL, High-density lipoprotein; TSH, Thyroid-stimulating hormone. 
Table 2: Comparison of different variables in patients with COVID-19 admitted to the ICU with non-ICU.

\begin{tabular}{|c|c|c|c|}
\hline Variable & $\begin{array}{l}\text { Non-ICU } \\
(\mathrm{n}=315)\end{array}$ & $\begin{array}{c}\text { ICU } \\
(\mathrm{n}=90)\end{array}$ & $p$-value \\
\hline Total bilirubin (mg/dL) & 1.32 & 2.29 & 0.014 \\
\hline Direct bilirubin (mg/dL) & 0.53 & 1.10 & 0.005 \\
\hline Age (year) & 60.80 & 61.81 & 0.604 \\
\hline AST (U/L) & 58.69 & 78.50 & 0.211 \\
\hline ALT (U/L) & 48.15 & 57.26 & 0.590 \\
\hline $\operatorname{ALP}(\mathrm{U} / \mathrm{L})$ & 286 & 295 & 0.854 \\
\hline Albumin (g/dL) & 3.64 & 3.25 & 0.001 \\
\hline PTT (second) & 27.89 & 33.38 & 0.003 \\
\hline PT (second) & 16.85 & 18.48 & 0.110 \\
\hline $\mathrm{pH}$ & 7.38 & 7.35 & 0.013 \\
\hline $\mathrm{HCO} 3(\mathrm{mEq} / \mathrm{L})$ & 24.40 & 21.43 & 0.000 \\
\hline Platelet (per $\mu \mathrm{L}$ ) & 204,855 & 200,826 & 0.741 \\
\hline $\mathrm{CRP}(\mathrm{mg} / \mathrm{L})$ & 52.66 & 71.73 & 0.000 \\
\hline FBS (mmol/L) & 125.9 & 173.0 & 0.002 \\
\hline Total cholesterol (mg/dL) & 118.37 & 132.38 & 0.324 \\
\hline Triglyceride (mg/dL) & 121.57 & 175.08 & 0.029 \\
\hline LDL (mg/dL) & 65.40 & 71.27 & 0.612 \\
\hline HDL (mg/dL) & 29.36 & 30.90 & 0.701 \\
\hline Fibrinogen(mg/dL) & 569 & 506 & 0.632 \\
\hline LDH (U/L) & 908 & 1160 & 0.054 \\
\hline $\mathrm{TSH}(\mathrm{mU} / \mathrm{L})$ & 2.38 & 1.78 & 0.647 \\
\hline Length of admission (day) & 5.15 & 10.37 & 0.000 \\
\hline
\end{tabular}

aminotransferases were mildly higher on admission in the ICU group and in those who died of COVID-19, but the difference was not statistically significant.

Also, in our study, liver function tests, such as total and direct bilirubin, PTT, PT, and albumin, showed a significant difference between those who were discharged and those who died. Likewise, Zhang and colleagues showed that patients with COVID-19 commonly had abnormal bilirubin and albumin levels, and the bilirubin level was significantly different between severe and mild cases, but the difference was not clinically significant. ${ }^{18}$ Also, Yadav and co-workers reviewed nine studies that included 2115 patients and showed that liver injury was associated with an increased risk of severity and mortality in patients with COVID-19. ${ }^{19}$

FBS and triglyceride levels were also significantly correlated with increased admission to the ICU. We think the reason is the association of severe COVID-19 with metabolic syndrome. Platelet counts have a critical role in predicting mortality in these patients; those who had died had lower platelet counts. As we know, a decrease in platelet counts could be an indicator of disseminated intravascular coagulopathy and a worse outcome.

Liver injury in COVID-19 is probably multifactorial: 1- viral infection in hepatocytes, 2- drug-induced liver injury, and 3- systemic inflammation caused by the cytokine storm or hypoxia. ${ }^{20}$ Liver tissue from an autopsied COVID-19 patient did not show any viral inclusions; more research is needed to clarify the mechanism of liver injury. ${ }^{21}$

Some limitations may have affected our results. We only enrolled patients who were admitted to hospitals; this could cause a selection bias.

The SARS-CoV-2 pandemic has rapidly spread around the globe, causing a public health and economic crisis. We still need more research as much is still unknown regarding this rapidly spreading disease. We recommend that 
physicians involved in the care of patients with COVID-19 pay closer attention to liver function tests, which can be a prominent feature of this disease, predicting serious clinical consequences.

\section{CONCLUSION}

Liver function tests, such as total and direct bilirubin, PTT, PT, and albumin, accurately predict mortality in patients with COVID-19. Moreover, FBS and triglyceride levels were also significantly correlated with increased admission in the ICU. Also, liver enzymes were only slightly increased in those who died or needed ICU admission and were not related to the fatty liver changes.

\section{ACKNOWLEDGMENTS}

This work was not supported by any funding.

\section{ETHICAL APPROVAL}

There is nothing to be declared.

\section{CONFLICT OF INTEREST}

The authors have none to declare.

\section{REFERENCES}

1. Zhu N, Zhang D, Wang W, Li X, Yang B, Song J, et al. A novel coronavirus from patients with pneumonia in China, 2019. $N$ Engl J Med 2020;382:727-33. doi: 10.1056/ NEJMoa2001017.

2. Sahafizadeh E, Sartoli S. Estimating the reproduction number of COVID-19 in Iran using epidemic modeling. medRxiv 2020.

3. Meters W. Total Coronavirus Cases in Iran. 2020; https:// www.worldometers.info/coronavirus/country/iran/. Accessed 12 May, 2020.

4. Holmes KV. SARS-associated coronavirus. $N$ Engl $J$ Med 2003;348:1948-51. doi: 10.1056/NEJMp030078.

5. Cui J, Li F, Shi ZL. Origin and evolution of pathogenic coronaviruses. Nat Rev Microbiol 2019;17:181-92. doi: 10.1038/s41579-018-0118-9.

6. Chen N, Zhou M, Dong X, Qu J, Gong F, Han Y, et al. Epidemiological and clinical characteristics of 99 cases of 2019 novel coronavirus pneumonia in Wuhan, China: a descriptive study. Lancet 2020;395:507-13. doi: 10.1016/ S0140-6736(20)30211-7.

7. Zu ZY, Jiang MD, Xu PP, Chen W, Ni QQ, Lu GM, et al. Coronavirus disease 2019 (COVID-19): a perspective from China. Radiology 2020;296:E15-E25. doi: 10.1148/radiol.2020200490.

8. Liu C, Jiang Z, Shao C, Zhang HG, Yue HM, Chen ZH, et al. Preliminary study of the relationship between novel coronavirus pneumonia and liver function damage: a multicenter study. Zhonghua Gan Zang Bing Za Zhi 2020;28:107-11. doi: 10.3760/cma.j.issn.1007-3418.2020.02.003.

9. Mantovani A, Beatrice G, Dalbeni A. Coronavirus disease 2019 and prevalence of chronic liver disease: A meta-analysis. Liver Int 2020;40:1316-20. doi: 10.1111/liv.14465.

10. Liu F, Long X, Zhang B, Zhang W, Chen X, Zhang Z. ACE2 Expression in Pancreas May Cause Pancreatic Damage After SARS-CoV-2 Infection. Clin Gastroenterol Hepatol 2020;18:2128-30. doi: 10.1016/j.cgh.2020.04.040.

11. Huang Y, Zhou H, Yang R, Xu Y, Feng X, Gong P. Clinical characteristics of 36 non-survivors with COVID-19 in Wuhan, China. medRxiv 2020. doi: 10.1101/2020.02.27.20029009.

12. Lai CC, Shih TP, Ko WC, Tang HJ, Hsueh PR. Severe acute respiratory syndrome coronavirus 2 (SARS-CoV-2) and corona virus disease-2019 (COVID-19): the epidemic and the challenges. Int J Antimicrob Agents 2020:105924. doi: 10.1016/j.ijantimicag.2020.105924.

13. Pickhardt PJ, Park SH, Hahn L, Lee SG, Bae KT, Yu ES. Specificity of unenhanced CT for non-invasive diagnosis of hepatic steatosis: implications for the investigation of the natural history of incidental steatosis. Eur Radiol 2012;22:107582. doi: 10.1007/s00330-011-2349-2.

14. Xu L, Liu J, Lu M, Yang D, Zheng X. Liver injury during highly $\mathrm{p}$ athogenic human coronavirus infections. Liver Int 2020;40:998-1004. doi: 10.1111/liv.14435.

15. Zhang C, Shi L, Wang FS. Liver injury in COVID-19: management and challenges. Lancet Gastroenterol Hepatol 2020;5:428-30. doi: 10.1016/S2468-1253(20)30057-1.

16. Guan Wj, Ni Zy, Hu Y, Liang WH, Ou CQ, He JX, et al. Clinical characteristics of coronavirus disease 2019 in China. N Engl J Med 2020;382:1708-20. doi: 10.1056/NEJMoa2002032.

17. Wang D, Hu B, Hu C, Zhu F, Liu X, Zhang J, et al. Clinical Characteristics of 138 Hospitalized Patients With 2019 Novel Coronavirus-Infected Pneumonia in Wuhan, China. JAMA 2020;323:1061-9. doi: 10.1001/jama.2020.1585.

18. Zhang Y, Zheng L, Liu L, Zhao M, Xiao J, Zhao Q. Liver impairment in COVID-19 patients: A retrospective analysis of 115 cases from a single centre in Wuhan city, China. Liver Int 2020;40:2095-2103. doi: 10.1111/liv.14455.

19. Yadav DK, Singh A, Zhang Q, Bai X, Zhng W, Yadav RK, et al. Involvement of liver in COVID-19: systematic review and meta-analysis. Gut 2021;70:807-9. doi: 10.1136/ gutjnl-2020-322072.

20. Xie H, Zhao J, Lian N, Lin S, Xie Q, Zhuo H. Clinical characteristics of non-ICU hospitalized patients with coronavirus disease 2019 and liver injury: A retrospective study. Liver Int 2020;40:1321-6. doi: 10.1111/liv.14449.

21. Hanley B, Lucas SB, Youd E, Swift B, Osborn M. Autopsy in suspected COVID-19 cases. J Clin Pathol 2020;73:239-42. doi: 10.1136/jclinpath-2020-206522. 